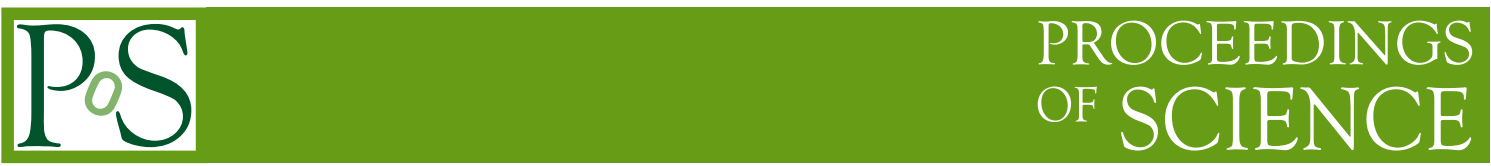

\title{
Recent Cross-Section Results from MicroBooNE
}

\section{Pip Hamilton, for the MicroBooNE collaboration*}

Syracuse University

E-mail: phhamiltesyr.edu

\begin{abstract}
MicroBooNE is a liquid argon time projection chamber located on the Booster Neutrino Beam at Fermilab. The large event rate and $3 \mathrm{~mm}$ wire spacing of the detector provide high-statistics, precise-resolution imaging of neutrino interactions leading to low-threshold, high-efficiency event reconstruction with full angular coverage. This provides an excellent environment to probe neutrino-argon interactions in the hundreds-of-MeV to few-GeV energy range, and to study the impact of nuclear effects through detailed measurements of hadronic final states. These proceedings present recent cross-section measurements of neutrino interactions in MicroBooNE, including inclusive charged-current interactions, neutral-pion production, and measurements of low-energy protons.
\end{abstract}

The 21st international workshop on neutrinos from accelerators (NuFact2019)

August 26 - August 31, 2019

Daegu, Korea

${ }^{*}$ Speaker. 


\section{Introduction}

Liquid Argon Time Projection Chambers (LArTPCs) are a detector technology with powerful applications in neutrino physics. LArTPC technology has been tested in experiments such as ICARUS [2] and ArgoNeuT [1], and will be deployed on a colossal scale to measure neutrino oscillations for the DUNE experiment [3].

MicroBooNE is the largest LArTPC currently in operation, with an active volume of 90 tons of liquid argon. This volume is read out by more than 8,000 wires that collect drifting charge (spaced $3 \mathrm{~mm}$ apart to give fine spatial resolution) and 328 " PMTs that collect scintillation light. The detector sits $470 \mathrm{~m}$ downstream from the Booster Neutrino Beam (BNB) dump, and has been collecting data from the BNB for 4 years (as well as off-axis neutrinos from the NuMI beam). This gives MicroBooNE a wealth of data that can be used to study neutrino-argon cross-sections. Some of the cross-sections recently produced by MicroBooNE are detailed here.

\section{MicroBooNE Cross-Section Results}

\subsection{Charged Current Inclusive}

The charged-current inclusive cross-section is the simplest and highest-statistics cross-section for MicroBooNE to measure, with a robustly defined final state. It provides a global test of neutrino interaction models, as it represents the sum of all charged-current interaction channels. It also has a direct bearing on the $v_{\mu}$ disappearance rate for DUNE.

There are three main challenges in trying to measure this cross-section in MicroBooNE: rejecting cosmic muons, identifying neutrino vertices, and reconstructing the neutrino energy. To mitigate cosmic contamination, the MicroBooNE charged current selection requires fully-contained muon tracks (identifying entering muons which stop via Michel decay). It also uses scintillation light from the argon to identify which tracks originating in the beam window, and reject those outside it. Vertexing is handled by the Pandora reconstruction suite [11], while energy reconstruction is done through multiple Coulomb scattering. The full selection is detailed in the recently published paper on this measurement [4]. With this selection, we extract a flux-integrated cross-section:

$$
\sigma=0.693 \pm 0.010 \text { (stat.) } \pm 0.165 \text { (syst.) } \times 10^{-38} \mathrm{~cm}^{2} .
$$

This result is shown in the context of world data in Figure 1. Using the same selection, we also extract a differential cross-section in the reconstructed muon momentum and angle: this is shown in slices of reconstructed muon momentum in Figure 2.

\subsection{Charged Current $\pi^{0}$}

Neutral current $\pi^{0}$ production is a major background for $v_{\mathrm{e}}$ appearance searches, which constitute one of MicroBooNE's main physics missions in searching for sterile neutrinos. Charged current $\pi^{0}$ production provides an easily measurable channel that helps constrain some of the same processes. It is also a neutrino interaction that has never before been studied on argon.

The recently published MicroBooNE charged current $\pi^{0}$ analysis [5] uses similar selection tools to those in the charged-current inclusive analysis, with the added condition of searching for 


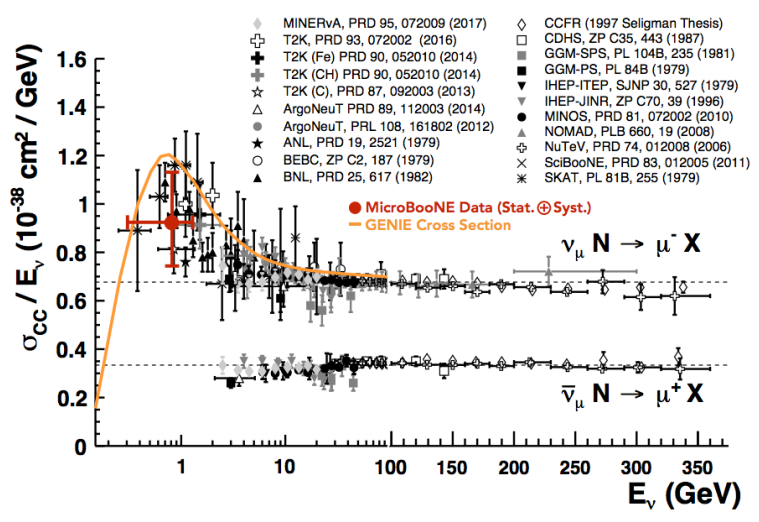

Figure 1: Flux-integrated $v_{\mu}$-Ar charged-current inclusive cross-section in the context of world data.
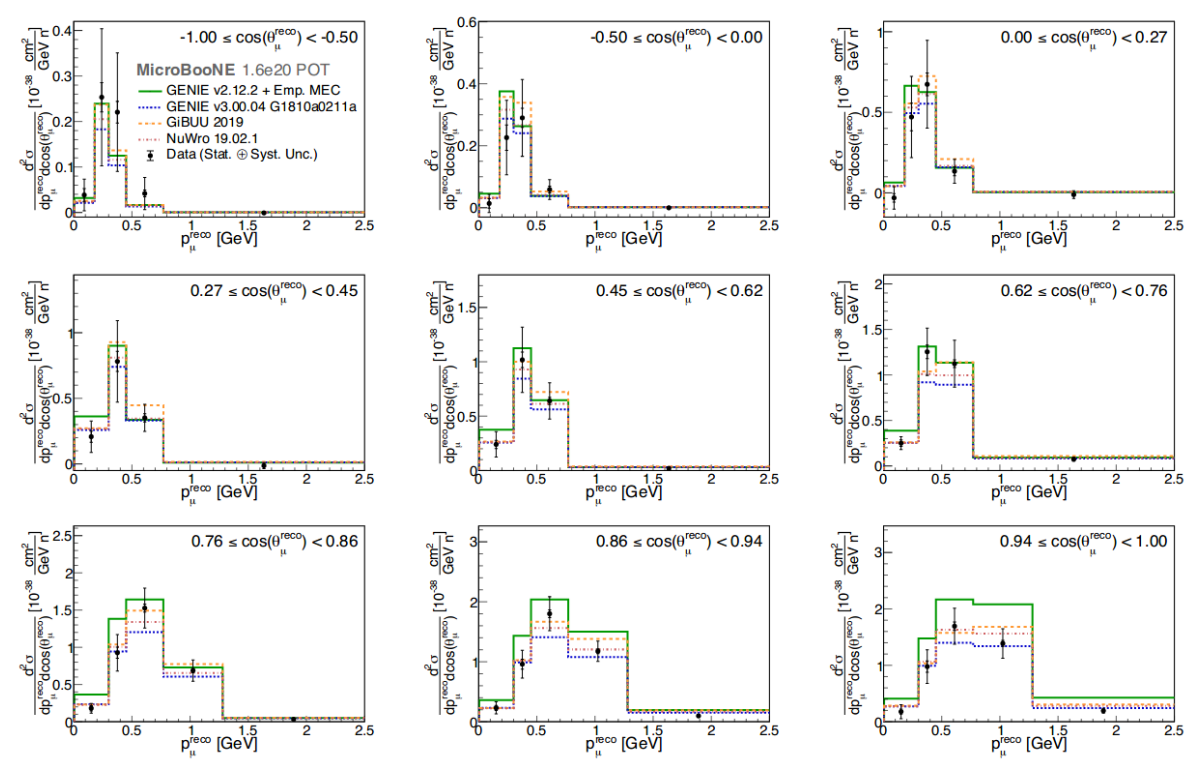

Figure 2: Differential cross-section as a function of $\cos \theta_{\mu}$, in slices of $p_{\mu}$, contrasted with predictions from three different Monte Carlo neutrino generators. The best agreement appears to be with GENIE v3, which has a local Fermi gas model and therefore a lower cross-section in the forward region.

an electromagnetic shower in the proximity of the neutrino vertex. Using this selection, we extract a flux-integrated cross-section:

$$
\sigma=1.9 \pm 0.2 \text { (stat.) } \pm 0.6 \text { (syst.) } \times 10^{-38} \mathrm{~cm}^{2} \text { per Ar nucleus. }
$$

This result is shown in comparison to the ANL [6] and MiniBooNE [7] measurements of this channel on deuterium and carbon in Figure 3.

\subsection{Charged Particle Multiplicity}

Measuring charged particle multiplicity provides an empirical test of the scattering models used in neutrino generators. There have been few measurements of this kind on argon, and with 


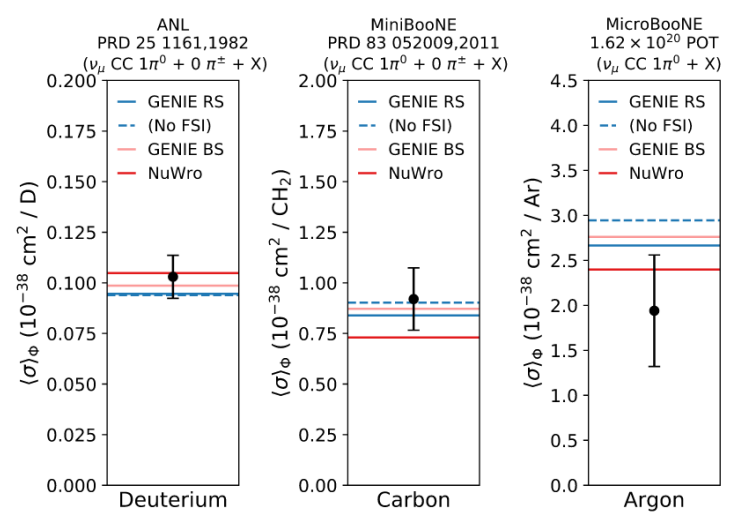

Figure 3: The flux-integrated charge-current $\pi^{0}$ cross-section on argon at MicroBooNE (right) compared to carbon at MiniBooNE (centre) and deuterium at ANL (left). The coloured lines show the predictions of various neutrino interaction models. The NuWro prediction seems most consistent with the MicroBooNE data.

its low tracking threshold MicroBooNE is well-positioned to make such a measurement. In our recently published paper [8], we produced a measurement of the relative event fraction in each bin of charged particle multiplicity, as shown in Figure 4.

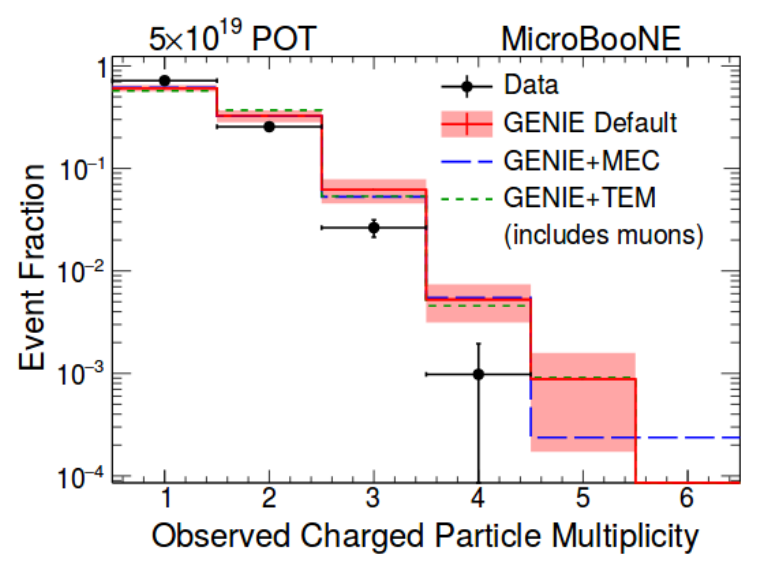

Figure 4: The distribution of the relative event fraction for multiplicities 1-6. The data is lower than the GENIE prediction in the high-multiplicity bins, and higher than GENIE in the multiplicity = 1 bin.

\subsection{Other Ongoing Analyses}

MicroBooNE's ongoing charged current n-proton analysis provides a direct measurement of ' $2 \mathrm{p} 2 \mathrm{~h}$ ' [9] processes in the nucleus, exploiting the low momentum threshold for proton reconstruction and the $4 \pi$ angular acceptance of the MicroBooNE TPC to further the work done by ArgoNeuT in selecting 'hammer' events [10]. The analysis in its current form is available as a public note [12].

MicroBooNE has also developed a selection for $v_{\mathrm{e}}$ from the NuMI beam. $v_{\mathrm{e}}$ appearance is a golden channel for SBN and DUNE, so there is a pressing need to understand the $v_{\mathrm{e}}$ cross-section on argon. With approximately 100 selected events, MicroBooNE has successfully selected the largest $v_{\mathrm{e}}$ interaction sample on argon to date. This analysis is also detailed in a public note [13]. 


\section{Conclusions}

MicroBooNE has published three neutrino interaction results, each of which provides an empirical test of our interaction models. Many other neutrino interaction measurements are currently underway using the MicroBooNE data. MicroBooNE's automated event reconstruction is now tested and mature enough to support these analyses, and is still growing more sophisticated. Once the full MicroBooNE dataset is unblinded, we expect the expanded statistics to allow us to make powerful measurements of rare and highly-specific cross-section channels.

\section{References}

[1] R. Guenette, arXiv:1110.0443 [physics.ins-det].

[2] F. Arneodo et al. [ICARUS Collaboration], hep-ex/0103008.

[3] B. Abi et al. [DUNE Collaboration], arXiv:1807.10334 [physics.ins-det].

[4] P. Abratenko et al. [MicroBooNE Collaboration], Phys. Rev. Lett. 123, no. 13, 131801 (2019) doi:10.1103/PhysRevLett.123.131801 [arXiv:1905.09694 [hep-ex]].

[5] C. Adams et al. [MicroBooNE Collaboration], Phys. Rev. D 99, no. 9, 091102 (2019) doi:10.1103/PhysRevD.99.091102 [arXiv:1811.02700 [hep-ex]].

[6] G. M. Radecky et al., Phys. Rev. D 25, no. 5, 1161-1173 (1982) doi:10.1103/PhysRevD.25.1161.

[7] A. Aguilar-Arevalo et al. [MiniBooNE Collaboration], Phys. Rev. D 83, no. 5, 052009 (2011) doi: 10.1103/PhysRevD.93.052009.

[8] C. Adams et al. [MicroBooNE Collaboration], Eur. Phys. J. C 79, no. 3, 248 (2019) doi:10.1140/epjc/s10052-019-6742-3 [arXiv:1805.06887 [hep-ex]].

[9] R. Gran, J. Nieves, F. Sanchez and M. J. Vicente Vacas, Phys. Rev. D 88, no. 11, 113007 (2013) doi:10.1103/PhysRevD.88.113007 [arXiv:1307.8105 [hep-ph]].

[10] L. B. Weinstein, O. Hen and E. Piasetzky, Phys. Rev. C 94, no. 4, 045501 (2016) doi:10.1103/PhysRevC.94.045501 [arXiv:1604.02482 [hep-ex]].

[11] R. Acciarri et al. [MicroBooNE Collaboration], Eur. Phys. J. C 78, no. 1, 82 (2018) doi:10.1140/epjc/s10052-017-5481-6 [arXiv:1708.03135 [hep-ex]].

[12] Public Note 1056, https://microboone.fnal.gov/public-notes/.

[13] Public Note 1054, https://microboone.fnal.gov/public-notes/. 